\title{
LA CONSULTORÍA FILOSÓFICA DE RAN LAHAV, OSCAR BRENIFIER Y ORA GRUENGARD: ¿APROXIMACIONES INCOMPATIBLES?
}

\author{
CARMEN ZAVALA \\ Universidad Nacional Federico Villarreal, Perú \\ Sociedad Peruana de Consejería Filosófica y Práctica Filosófica \\ carmen@zavala.de
}

RECIBIDO: 12 DE DICIEMBRE DE 2009

ACEPTADO: 20 DE ABRIL DE 2010

Resumen: En el presente artículo pretendo mostrar a través de un análisis de mi propio trabajo práctico, que aproximaciones en el trabajo de la consultoría filosófica tan distintas como las de Ran Lahav, Oscar Brenifier y Ora Gruengard no son en realidad tan incompatibles como ellos mismos consideran que son.

Para ello comentaré extractos de una sesión de consultoría filosófica mía filmada hace un tiempo atrás, indicando las coincidencias con estos filósofos, que motivaron o inspiraron algunos de los pasos que llevé a cabo en esta sesión. Asimismo trataré de absolver algunas de las posibles objeciones a las comparaciones propuestas.

Mi propósito entonces es mostrar en base a un ejemplo práctico, cómo las reflexiones y discusiones sobre los diferentes aspectos de la consultoría filosófica de filósofos prácticos de diferentes aproximaciones, pueden servir para enriquecer la práctica filosófica y abrir nuevas perspectivas para su desarrollo, en vez de usarse para excluirse mutuamente.

Palabras clave: consultoría filosófica, racionalidad, actitud filosófica

\begin{abstract}
In this paper I mean to show, through an analysis of my own practical work that approaches of philosophical counseling as different as those of Ran Lahav, Oscar Brenifier and Ora Gruengard are not really that incompatible as they themselves consider them to be.

To show this I am going to comment a videotaped session of philosophical counseling that I made to a counselee some time ago and I will show the coincidences with these philosophers, who motivated or inspired some of the steps I took. I also will try to respond to some of the possible objections to the comparisons that I am proposing.

My purpose is to show through a practical example, how the reflections and discussions of different aspects of philosophical counseling of philosophical counselors with different approaches can help to enrich philosophical practice and open up new perspectives for its development, instead of excluding each other.
\end{abstract}

Keywords: philosophical counselling, rationality, philosophical attitude. 


\section{Introducción}

En el presente artículo pretendo mostrar a través de un análisis de mi propio trabajo práctico, que aproximaciones en el trabajo de la consultoría filosófica tan distintas como las de Ran Lahav, Oscar Brenifier y Ora Gruengard no son en realidad tan incompatibles como ellos mismos consideran que son.

Para ello comentaré extractos de una sesión de consultoría filosófica mía filmada hace un tiempo atrás, indicando las coincidencias con estos filósofos, que motivaron o inspiraron algunos de los pasos que llevé a cabo en esta sesión. Asimismo trataré de absolver algunas de las posibles objeciones a las comparaciones propuestas. El consultante otorgó el permiso para que se publicaran estos extractos de la trascripción de la sesión en el presente artículo.

El argumento principal de estos filósofos, a favor de que sus aproximaciones no son compatibles, es que parten de concepciones distintas sobre qué es la práctica filosófica en general y la consultoría filosófica en particular. Mi argumento principal para afirmar lo contrario es que diferentes concepciones y motivaciones suelen confluir muchas veces en su práctica y propósitos concretos. En ese sentido me inspiro en Marx cuando dice: "Los filósofos no han hecho más que interpretar de diversos modos el mundo, pero de lo que se trata es de transformarlo." , que interpreto como que podemos teorizar mucho sobre qué es la filosofía y la práctica filosófica, pero de lo que se trata principalmente es de la práctica concreta, la cual, por una parte, influye en la realidad, por el trabajo de reflexión filosófica con personas concretas como, por otra parte, en la visión de la realidad sobre la práctica filosófica, pues crea un marco de experiencias nuevas que permiten avanzar en la teoría tanto sobre la practica filosófica en particular, como sobre la filosofía en general.

Y así resulta que en la práctica concreta el consejero filosófico se tiene que adaptar en mayor o menor grado a las circunstancias prácticas concretas del mundo del consultante, más que en sus presupuestos

\footnotetext{
${ }^{1}$ MARX, Karl: "Tesis sobre Feuerbach", Tesis 11 en: Obras escogidas, T I, Moscú: Editorial Progreso, 1976, p. 10
} 
teóricos, para que la consultoría filosófica logre su propósito de elevar al consultante a la reflexión filosófica sobre los problemas que lo ocupan.

En el marco de esta dialéctica entre teoría y praxis, es que Gerd Achenbach afirma que el primer principio de la práctica filosófica es que a cada consultante hay que abordarlo según su forma muy particular de ser $^{2}$.

Mi propósito entonces es mostrar en base a un ejemplo práctico, cómo las reflexiones y discusiones sobre los diferentes aspectos de la consultoría filosófica de filósofos prácticos de diferentes aproximaciones, pueden servir para enriquecer la práctica filosófica y abrir nuevas perspectivas para su desarrollo, en vez de usarse para excluirse o ningunearse mutuamente. Asimismo aprovecho este artículo para reunir extractos de materiales sobre consultoría filosófica de Brenifier, Lahav,

\footnotetext{
2 ACHENBACH, Gerd: "Al centro del la Práctica Filosófica" (Zur Mitte der philosophischen Praxis, traducción propia), Conferencia de apertura del II Congreso Internacional de Práctica Filosófica en Amersfoort/Leusden, Holanda, 25 de agosto de 1996.

"Voy a explicar esto (el primer principio de la Práctica Filosófica) brevemente con un ejemplo: Acá aparece uno (un consultante) impresionado por los pasos sólidos aparentemente impecables de la argumentación lógica, que está fascinado por el carácter necesario de las consecuencias de nuestro raciocinio, que con gusto se somete a las conclusiones que le garantizan lo correcto. Bien - en ese caso, argumente con él, y en el mejor de los casos experimentará los que con toda razón afirma Marvin Minsky: 'El pensar influye en nuestros pensamientos'.

Pero sólo tiene razón en este caso. Pues por allí aparece otro consultante, al que aquella corrección en el pensar lógico ( justamente tan apreciada recién por aquel primer consultante) le da miedo, en tanto la considera señal de frialdad y de una intelectualidad inauténtica, que oculta un afán de prepotencia y que frente a esta aproximación emprende la retirada y se retrae en su interior, y que experimenta como su conocido desinterés en el trabajo de construcción argumentativa del juicio se transforma en odio y profundo desprecio por $s u$ aporte bien intencionado (su aporte como consejero filosófico). También quiero comentar este caso con una frase prestada, una frase del Kuduz: "Cuando no podemos convencer un cerebro, no hemos sido capaces de convertir un corazón." Y para aquel que en el caso de una tal persona, de todos modos tratase de convencer su cerebro pasando por su corazón, cabría decirle a través de Paul Feyerabend, lo que se le ha pasado: 'De qué sirve un argumento, que deje fría a la gente?'

A un tercero (a un tercer consultante) aquello sobre lo que nos cuenta que le preocupa, que lo tiene envuelto, paralizado, que no lo deja avanzar, no puede ser aclarado de otro modo que haciendo encajar todos los pedazos y fragmentos de su vida en una relación casi mítica, en todo caso transmisible juntándolos en una historia clara. Recién entonces él ve, lo que le concierne, logra abarcar con la mirada el entretejido de su destino, vislumbra el sentido de los sucesos que había estado buscando o que en todo caso de alguna manera echaba de menos; sucesos que hasta entonces le habían sido extraños e incomprensibles y que ahora tal vez pueda reconocer como una tarea que le ha sido asignada y así pueda aprender a soportar a su manera la pesada carga, que 'la vida' le ha adjudicado."
} 
Gruengard y Achenbach, lo cual puede facilitar la discusión futura sobre la práctica filosófica en español.

\section{Sobre la relación del consejero filosófico con el consultante}

En el presente caso el consultante es un profesional que hace más de un año ha estado viniendo con cierta periodicidad a las sesiones de consultoría filosófica, al cual llamaremos Eduardo para mantener su privacidad. La sesión de aproximadamente una hora empezó de la siguiente manera.

Eduardo: Tengo 3 preguntas que podríamos tratar hoy. La primera es: ¿Se me fue la vida? Tenía esa sensación de que ya estoy un poco tarde. La otra pregunta es: ¿Tengo que contarle a todo el mundo que soy gay? A veces pienso que sería más fácil no mencionarlo. Me evitaría una serie de problemas.

$\mathrm{Ah}$ ! Y hay una pregunta más, ¿debo seguir asistiendo al Café Filosófico?¿Cuál podría ser más interesante?

C.F $\quad$ Y a ti ¿cuál te parece más interesante?

En realidad a mí me parecía más interesante la primera pregunta. La tercera pregunta de alguna manera me involucraba personalmente en el sentido de que yo junto a otros colegas filósofos somos los que dirigimos el Café Filosófico al que hace mención, y en ese sentido, yo sentía que el consultante induciría a cuestionar nuestro proyecto de práctica filosófica y a mí, lo cual me mortificaba, porque probablemente entrarían en juego factores subjetivos y la objetividad de ambas partes podría hacerse más difícil, aunque no imposible. Con respecto a este tema, tanto Oscar Brenifier $^{3}$ como Ran Lahav ${ }^{4}$, presuponen que en el momento de la

\footnotetext{
${ }^{3}$ Brenifier pone bastante énfasis en la separación del "hombre" y el "filósofo" a la hora de empezar una sesión de consultoría filosófica: "Para efectuar los procedimientos cognoscitivos (en la consultoría filosófica) se necesita estar en un cierto estado mental, tener una específica clase de actitud, hecha de distancia y de perspectiva crítica. Esta actitud es muy exigente, conoce muchos obstáculos. La sinceridad por ejemplo es un obstáculo para esta actitud, también la buena conciencia y la subjetividad, que tiene que renunciar a su estricto dominio sobre la mente. Más radicalmente, los principios morales, los postulados cognitivos y las necesidades psicológicas que nos guían en la vida tienen que ponerse entre paréntesis, someterse a la dura crítica, incluso ser rechazados, lo que por supuesto no pasa de manera natural ya que produce dolor y angustia, aunque uno sea capaz de tomar
} 
consultoría filosófica el consejero filosófico es filósofo ante todo y debe liberarse de sus actitudes y perspectivas subjetivas, dejando de ser el individuo humano que es durante la sesión. En otras palabras hay que ser lo más objetivo posible. Este es uno de los factores que se considera que constituye el profesionalismo en la consultoría filosófica: el olvidarse de las opiniones propias, y evitar así caer en la tentación de opinar, explicarse o dar consejos, en vez de centrarse en el discurso, las actitudes y las eventuales ansiedades del consultante.

A pesar de que tanto Brenifier como Lahav tienen una concepción distinta de qué es una "actitud filosófica", ambos sostienen que se trata de una actitud frente a la vida en su totalidad y no se limita a la sesión de práctica filosófica. Manejar la consultoría sin involucrarse parece muy simple pero llevarlo a la práctica no siempre lo es, sobre todo si el consultante pretende involucrar al consejero filosófico o a la interacción con el consejero filosófico como objeto de análisis. Esta dificultad es notoria por ejemplo en algunas sesiones públicas videofilmadas con Oscar Brenifier, en las que la consultante no plantea un problema propio, sino que cuestiona al consejero filosófico y su quehacer ${ }^{5}$.

distancia con respecto de sí mismo. Dividirse a sí mismo, como Hegel sugiere, como condición de un pensamiento real, como condición de la conciencia. Y para poder completar semejante cambio de actitud, hay que morir a uno mismo, abandonar, incluso de manera momentánea, lo que es más querido, la idea prudente, la emoción prudente." BRENIFIER, Oscar: "Filosofar es dejar de vivir", disponible on-line en

www.brenifier.com/espanol/filosofar_es_dejar_de_vivir.htm (último acceso 30 de noviembre de 2009).

${ }^{4}$ Si bien Lahav también hace la distinción entre la persona como hombre y la persona en su calidad de filósofo, considera que la persona del filósofo debe aspirar a ser filósofo no sólo durante la sesión de consultoría filosófica u otros quehaceres filosóficos, y en ese sentido debe aspirar a mantener una actitud filosófica en todos los momentos de su vida: "En tanto filó-sofo no pienso ni hablo solo en nombre de mi propio pequeño yo. No siento, ni actúo desde mi ego centrado en mí mismo, desde la prisión de mis presupuestos, desde mis juegos sicológicos y sociales. En vez de eso, dejo que una forma de entendimiento mayor hable dentro de mí. En otras palabras, le doy voz a esas partes de mi ser que normalmente están reprimidas y marginadas por los juegos sociales, por mis necesidades sicológicas, por mi agenda personal y prejuicios culturales. Aspiro a llegar a tener una mayor conciencia, que permita a otras partes de mi realidad, hablar a través mío, y por lo tanto, permitir que un rango mayor de comprensión actúe en mi vida. LAHAV, Ran: "Desarrollando la Sensibilidad Filosófica", disponible on-line en www.trans-sophia.net/115845/21--La-sensibilidad (último acceso 30 de noviembre de 2009)

${ }^{5}$ Consultoría Filosófica que Brenifier le hace a la profesora Lumena, y en la que ésta no plantea un problema suyo, sino que más bien trata de demostrar que el método mayéutico que Brenifier usa es limitado porque "El discurso racional no puede llegar a ser un instrumento para abordar todos los problemas del hombre" video disponible online en BRENIFIER, Oscar: "El discurso racional no 
En este caso sin embargo, Eduardo eligió la primera pregunta y por lo tanto, inicialmente, no surgió la situación comentada.

\section{Sobre el reconocimiento del problema del consultante, la exploración de su vocabulario y la precisión de sus conceptos}

Eduardo: Esta pregunta de si “ ¿Se me fue la vida?” Me parece más interesante porque, es una sensación, más que una idea.

C.F.: ¿Por eso es más interesante? ¿Porque es una sensación?

Con esta pregunta trataba de que el consultante piense en lo que acaba de decir, y lo confirme, lo precise o lo descarte. Sobre la necesidad de este paso estarían de acuerdo tanto Brenifier y Lahav, como Ora Gruengard ${ }^{6}$. En el caso de Brenifier ${ }^{7}$ esta pregunta forzaría a Eduardo a asumir como verdadera la extraña proposición: "Lo importante son las sensaciones, no las ideas" y sus consecuencias. Y a partir de allí se podría, tal vez, empezar a trabajar en dicotomías, como por ejemplo, sensación vs. razón. Gruengard coincide con Brenifier en la necesidad del trabajo con oposiciones que reflejan dilemas filosóficos ${ }^{8}$, pero ella no llega a estas

puede llegar a ser un instrumento para abordar todos los problemas del hombre" disponible on-line en : http://www.brenifier.com/espanol/videos/el_discurso_racional_no_puede_llegar.htm (último acceso 30 de noviembre de 2009)

${ }^{6}$ Hacer precisar a la consultante el uso que del significado de las palabras en los diferentes juegos del lenguaje "es algo que le debo a los filósofos analíticos". GRUENGARD, Ora: "Ora Gruengard: Consultoría Filosófica (muestra)" disponible on-line en en video.google.de/videoplay?docid=6060796800723049099\&\# (último acceso 30 de noviembre de 2009)

7 "El orientador filosófico adquiere, pues, en esta fase un papel esencial, que consiste principalmente en subrayar aquello que ha sido dicho, para que las elecciones realizadas y sus implicaciones no pasen desapercibidas. Podrá incluso insistir, pidiéndole al sujeto que asuma las elecciones que acaba de expresar. Sin embargo, deberá evitar hacer cualquier comentario y evitará plantear ciertas preguntas complementarias si entrevé algún tipo de problema o de inconsecuencias en el discurso que acaba de ser articulado. Lo importante de esta parte del ejercicio consiste en conducir al sujeto para que evalúe libremente las implicaciones de sus posturas, para que entrevea aquello que su pensamiento oculta de sí mismo." BRENIFIER, Oscar:"La Consulta Filosófica - Los Principios”, disponible on-line en www.brenifier.com/espanol/orienta_principios.htm (último acceso 30 de noviembre de 2009)

8 "Mi "consultoría" filosófica está basada en que la "deconstrucción" de los problemas del consultante en grupos de oposiciones y en reconstruirlos como dilemas filosóficos que puedan ayudarlo, cuando se adapten al mundo del consultante para manejarlos de una manera constructiva y 
oposiciones forzando al consultante a través del preguntar mayéutico, como haría Brenifier. Más bien en la primera fase de reconocimiento y exploración del mundo y el vocabulario del consultante la dinámica que Gruengard sigue es bastante similar a la de Lahav ${ }^{9}$, fomentando ambos que en un primer momento la persona se explaye y permita darse a conocer y plantear lo que le preocupa con el mayor detalle posible. Lahav $^{10}$ por su parte, precisa que el repreguntar es importante porque él considera que es necesario determinar el vocabulario con el que cada persona describe su perímetro ${ }^{11}$, y además afirma que, para ello es necesario fijarse en los detalles extraños de lo que la persona hace o dice $^{12}$. Y en este caso resulta extraño, por lo menos a primera vista, que

reparadora." GRUENGARD, Ora, "Conocimiento y Diálogo en la Consejería Filosófica", Conferencia presentada en el "I Encuentro de Práctica Filosófica en el Perú" información disponible on-line en http://www.redfilosofica.de/practicafilosoficainternacional/

${ }^{9}$ Esto se puede observar al confrontar las partes iniciales de las sesiones de consultoría filosófica videograbadas de Lahav y Gruengard

LAHAV, Ran, "Ran Lahav en Consultoría Filosófica con Carmen (Inglés con subtítulos en español)" disponible on-line en http://www.trans-sophia.net/115845/VIDEO--Ran-Lahav-enConsultaria-Filosofica-con-Carmen (último acceso 30 de noviembre de 2009) y GRUENGARD, Ora, video citado.

10 "Primero, es importante preguntarnos: ¿Cuales son los conceptos principales en los perímetros de una persona? Estos conceptos pueden ser vistos como los ladrillos de construcción con los que se construye el perímetro, o como el VOCABULARIO con el que puede ser descrito. Un vocabulario es solo una lista no organizada de conceptos. Por eso, una segunda pregunta importante es: ¿Cuál es la estructura de estos conceptos? Para responder esta pregunta necesitamos hacer un análisis filosófico: determinar qué conceptos son centrales y cuáles son periféricos, cómo se relacionan los diferentes conceptos entre ellos, y cuál es el significado exacto de cada uno de ellos. Muchas veces un análisis así revela que la aparente superficie oculta una red muy diferente de ideas.

El resultado de este análisis es un MAPA CONCEPTUAL que muestra cómo los conceptos más importantes están organizados en el perímetro de la persona. El mapa representa, en otras palabras, la estructura del mundo de la persona. LAHAV, Ran, "Curso en Práctica Filosófica" disponible on-line en http://www.trans-sophia.net/115845/7--E-La-exploraci-n-del-per-metro (último acceso 30 de noviembre de 2009)

${ }^{11}$ El concepto de "perímetro" lo define Lahav como: "el mundo tal como lo entiendo yo, como lo experimento, como interactúo con él. (...) Es, como diría Platón, mi caverna. Incluye mis experiencias usuales, mis reacciones y comportamientos usuales, mis emociones y actitudes. (...)Un perímetro es una forma de limitación. Determina los tipos de historias que pueden ocurrir en mi mundo, y los roles que pueda jugar en ellas. Determina que sea probable que algunas situaciones vuelvan a ocurrir una y otra vez en mi vida." LAHAV, Ran: "El concepto de perímetro" disponible on-line en http://www.trans-sophia.net/115845/1--El-Concepto-de-Per-metro (último acceso 30 de noviembre de 2009)

12 "El consultor siempre está atento a detalles peculiares, que llaman la atención en la historia del consultado. Un detalle peculiar puede ser indicio de que algo interesante está pasando allí. Porque si lo que consultado hiciese fuese lo que todas las demás personas hacen, entonces no habría nada 
alguien afirme que una pregunta es importante porque expresa una sensación. Por ello también me pareció pertinente pedirle al consultante que aclare el concepto de sensación como algo interesante.

Eduardo: Lo que pasa es que para las otras dos preguntas tengo propuestas de respuesta, pero no para la primera

C.F.: $\quad$ O sea que, ¿lo que hace interesante una pregunta es que no haya ni siquiera una propuesta de respuesta?

Eduardo: Las mejores preguntas son las que no tienen respuestas.

Como todavía estábamos en la fase de reconocimiento del problema y de precisión de cómo el consultante usa los términos volví a la pregunta inicial.

C.F.: ¿Y por qué te parece que esa pregunta “¿se me fue la vida?” es una sensación?

Eduardo: Es una sensación que he tenido esta semana.... Sensación porque..... Bueno, es una idea, no es una sensación, o sea, es como si fuese una explicación para una serie de cosas que me han pasando

C.F.: $\quad$ O sea: ¿es una respuesta?

Eduardo: Podría ser una respuesta más que una pregunta. La pregunta podría ser entonces: ¿cómo regresar a la vida? en caso de que se pueda... ¿cómo hacerla volver?

Notamos acá la importancia de dejar que la persona se exprese libremente. La palabra "sensación" para el consultante no se refiere a una experiencia sensible ni interna, ni externa, como yo había pensado en un primer momento, sino que más bien se refiere a una "corazonada". Ante este hecho siento que debo dejar hablar más a Eduardo para que precise su preocupación y familiarizarme un poco más con el vocabulario que usa para ello.

interesante que decir al respecto. De modo que un detalle interesante puede ser la clave hacia un patrón interesante. "LAHAV, Ran, "Un video: Ran Lahav en consultoría Filosófica con Carmen" disponible on-line en http://www.trans-sophia.net/115845/VIDEO--Ran-Lahav-en-ConsultariaFilosofica-con-Carmen (último acceso 30 de noviembre de 2009) 
C.F.: $\quad$ Y en qué sentido dices que se ha ido (tu vida)?

Eduardo: Bueno, creo que tiene que ver con la edad que tengo. Quizás, a la edad que tengo debería haber hecho más cosas.

C.F.: ¿Cómo cuáles?

Eduardo: Debería tener un trabajo fijo. No tengo un trabajo fijo. No estoy encaminado profesionalmente. ....Hay muchas cosas que estoy comenzando... Se supone que debería haberlas comenzado a los 20 o 25 años... La pregunta es: ¿Es igual comenzar a los 35 que comenzar a los 20 ? Pienso que no es igual....

C.F.: $\quad$ Es interesante que digas que es necesario tener un trabajo fijo a cierta edad, en una época en la que justamente todo el mundo está perdiendo su trabajo, por la crisis. Serían todos ... como adolescentes..o jóvenes...

La idea con este comentario es discernir entre un eventual problema personal y un problema social. Concuerdo acá con Gruengard en que en muchos casos los problemas planteados por el consultante no necesariamente tienen que ver con trabas sicológicas o inhabilidades del consultante, sino que se presentan por situaciones de la realidad concreta externa sobre la que el consultante poco o nada puede influir, tales como podrían ser el desempleo, la enfermedad de algún familiar cercano, la vejez, una sociedad autoritaria, etc. ${ }^{13}$. Así por ejemplo el desempleo, no es en principio un problema causado por el individuo, pero si el consultante considera que sí lo es, habrá que ver qué otros factores y circunstancias están en juego que hacen, que en este caso especial, el consultante considere que es un problema causado por su propia deficiencia.

\footnotetext{
13 "Estar "atascado" en problemas, confusiones, conflictos y contradicciones no necesariamente es un asunto de incapacidad de ver las cosas bajo la "luz natural", ya sea que por "luz" se entienda el regalo de los dioses para algunos pocos elegidos o un asunto de "sentido común"normal. La "ceguera" a las aproximaciones alternativas no es ni un índice de maneras "pecaminosas" o "no-espirituales" de pensar, ni es un castigo por los pecados como algunos guías religiosos y pseudo religiosos aun tratan de convencernos. Tampoco es un asunto de pereza mental, asociaciones "no naturales" o hábitos "disfuncionales" como algunos filósofos y los psicólogos que los siguen, han tratado de enseñarnos al menos, desde los tiempos de Locke. (...)Y eso significa que el presupuesto de los psicólogos de que la razón por la que alguien se queda "atascado" en un problema está relacionada con "fijaciones" en modos de pensar "infantiles" o "pueriles", es dudosa, y solo puede explicar un pequeño número de casos. Es más, los psicólogos mismos están "atascados". No necesariamente en sus propias fijaciones infantiles, que ellos admiten, sino en sus desacuerdos sobre la naturaleza de lo "infantil" y lo "maduro". Muchos de ellos no son conscientes de que esos son desacuerdos filosóficos e imaginan que es un asunto ya sea de conocimiento científico o de interpretación según el gusto de cada quien." GRUENGARD, Ora, “Conocimiento y Diálogo en la Consejería Filosófica”, ob.cit.
} 
Eduardo: Eso significaría que el problema no es solo mío, sino que muchos tienen ese problema...

C.F.: $\quad$ Es un problema. El asunto es que tú lo has relacionado con la edad...

Eduardo: Sí, eso estoy pensando.... Creo que la pregunta básica sería: ¿no seré muy viejo para muchas cosas que quiero hacer?

C.F.: ¿Como cuáles?

Eduardo: Para muchas cosas de las que hemos estado discutiendo en los filoanálisis, como viajar, cambiar de ideas, buscar trabajo, cambiar mis relaciones, cambiar un poco mi for.., adaptarme...., esa clase de cosas.. ¿Hace alguna diferencia tener cierta edad?

C.F.: $\quad$ Voy a enumerar las cosas que has dicho que quieres hacer, aparentemente, ¿no?: viajar, buscar trabajo, buscar relaciones de pareja, adaptarte...¿a la sociedad?

Eduardo: Sí, adaptarme a la sociedad.

C.F.: ¿Esas son cosas que están relacionadas a la edad?

Eduardo: Sí, los jóvenes son los que hacen todas esas cosas con éxito. La pregunta es: Si yo he deseado siempre eso ¿por qué no lo he hecho?

Varios hilos han quedado sueltos y finalmente el consultante ha optado por reformular su pregunta inicial. Los hilos que quedaron sueltos se retomarán a lo largo de la discusión. Brenifier recomendaría tal vez aprovechar todas las contradicciones en el momento mismo en que se evidencian, para trabajar y dejando así la menor cantidad de cables sueltos posibles, para más tarde. En cualquiera de los casos, es deseable apuntar por escrito lo más resaltante sobre el consultado y una que otra frase o actitud insólita, sobre todo si se tiene mala memoria. También en este punto hay acuerdo entre los filósofos mencionados.

C.F. La pregunta entonces es: ¿Por qué no lo has hecho?

Eduardo: Sí, la pregunta tal vez sea ¿por qué no lo he hecho?

La pregunta se está modificando. Aparentemente todavía no está claro para el propio consultante qué es lo que realmente le preocupa. Tanto Ran Lahav como Ora Gruengard sugieren que es deseable iniciar la consultoría con un tiempo inicial de exploración en el pensamiento del consultante, a diferencia de Oscar Brenifier que más bien sugiere que en 
estas reformulaciones de la pregunta y del problema en general, se le estaría dejando espacio al consultante para presentarse como quiere que lo vean y ocultar lo que realmente le problematiza y no desea tratar, y que por lo tanto se estaría entorpeciendo el ejercicio del pensar.

En el caso del consultante Eduardo, a pesar de que la problematización de la edad era un tema planteado por primera vez por él, yo ya estaba familiarizada, en parte, con su vocabulario, por lo que el periodo de "exploración", podría teóricamente acortarse y se podría saltar de frente a una dinámica socrática de forzamiento del preguntar y el responder como el planteado por Oscar Brenifier. Sin embargo, opté por un preguntar y responder que permite un margen mucho más amplio en la respuesta, porque el forzar la pregunta en determinada dirección como propone Brenifier puede eventualmente forzar al consultante a pensar y responder lo que realmente piensa y revelar sus contradicciones como Brenifier sugiere, pero también es posible que, siendo el consultante ya de alguna manera consciente de sus contradicciones, más bien se pierdan matices importantes en el camino y desarrollo de su reflexión. Aquí quiero rescatar el primer principio de la práctica filosófica de Achenbach, mencionado más arriba, según el cuál es deseable abordar a cada consultante según su forma muy particular de $\operatorname{ser}^{14}$ y por otra parte para que haya un verdadero diálogo también es deseable escuchar al consultante para saber cómo él quiere ser visto y ser entendido por los demás. ${ }^{15}$

En el presente caso, en tanto Eduardo es justamente una persona fascinada por la argumentación racional y lógica, mi aproximación para con él es principalmente argumentativa, y hago notar las contradicciones e incoherencias en su discurso cuando estas se dan, para tratar de

\footnotetext{
${ }^{14}$ ACHENBACH, Gerd, ob.cit.

15 "Lo que me dijo (el consultante), no es para mí solo un motivo, para decir ahora algo por mi parte, sino que ante todo, y mientras estoy siendo el que está escuchando al otro - trato de entender, cómo es que se está tratando de presentar para mí, cómo es que desea ser entendido. Es decir, entender cómo es que él mismo se comprende. Pero esto no "está sobreentendido", como se dice, y de ninguna manera hay que suponer que el modo como él se da a entender corresponde al mío, es decir, a como yo me hago entender. De modo que no hay que suponer que pudiese ser posible una comprensión más o menos "directa". ACHENBACH, Gerd, "El arte de saber conversar", Ponencia para el 20 aniversario de la "Sociedad para la conducción de conversaciones en idiolecto" (GIG), Würzburg, 5 de mayo del 2005 accesible en http://www.achenbach-pp.de/cont/vortraege_pp.asp (último acceso 30 de noviembre de 2009)
} 
desentrañar sus presupuestos y consecuencias lógicas. Como habíamos visto, Achenbach señala en el primer texto mencionado, que en muchos casos la aproximación argumentativa podría producir rechazo en el consultante, y esto es algo que sucede muy frecuentemente con la aproximación de Oscar Brenifier. Sin embargo en otros muchos casos decía Achenbach -como en el caso presente sugiero yo- ésta es la aproximación que al consultante le parece más enriquecedora y a través de la cual siente que se lo respeta como interlocutor, en busca de respuestas.

Planteadas de alguna manera las preguntas: ¿Por qué no he hecho lo que hubiera querido hacer?, y ¿puedo hacer la cosas que quiero hacer? y ¿cómo podría hacerlo? Proseguimos en la indagación.

Más o menos 20 minutos después en la sesión llegamos al punto que viene a continuación.

\section{Sobre prácticas similares a partir de concepciones filosóficas diferentes}

C.F.: Tú ves personas que, según tú, tienen todo, pero viven descontentas.

Eduardo: Son personas que han hecho un diagnóstico sobre qué está mal en sus vidas. Han hecho un esfuerzo sobrehumano y han cambiado las cosas que estaban mal y las han sustituido por cosas que están bien, y siguen descontentas... es un asunto muy... es decir, ya no depende de nada de afuera.

C.F.: $\quad$ En cambio en tú caso (según lo que vinimos conversando) tú consideras que es objetivo todo lo que te sucede, estas limitaciones son objetivas. Y tú no has hecho nada para cambiarlas tampoco, por que no has podido, o que se yo. Entonces tú estás satisfecho porque has visto algo objetivo que no has podido cambiar, mientras que en el caso de los otros, obviamente es algo subjetivo - en el caso de las personas que tú estás diciendo. Pero qué pasaría, si hay un tercer grupo de personas que escuchan: "Acá está Eduardo, profesional, hace una serie de cosas, participa de una serie de actividades, tiene su vida económica resuelta, se las pasa entre reuniones científicas e intelectuales, de un lado a otro, no tiene mayores preocupaciones y anda lamentándose de su vida y queriendo ser otra persona" - y considerarían todo lo que tú 
haces como un drama inventado... O sea, así como tú hablabas de esos otros que a pesar de tenerlo todo viven insatisfechos.

Antes de dar otro paso resumo lo que el consultante había dicho y lo enfrento a una perspectiva diferente a su situación concreta. Esto tiene como objetivo, por una parte, relacionar la discusión teórica con su situación concreta, y por otra parte, poner en discusión la posibilidad de una perspectiva diferente a su visión de las cosas. Es un paso que he tomado de Brenifier quien lo utiliza con bastante frecuencia ${ }^{16}$.

Eduardo: Bueno sí hay todo tipo de gente con todo tipo de opiniones. Pero la pregunta sería: ¿qué tan justo sería lo que dicen? Eso es lo que podemos examinar ahora. Puede ser que tenga algo de cierto...Pero antes de continuar, yo quería decir: Yo no estoy seguro de que se trate de una situación exterior. Justamente la duda o sospecha de que sea algo subjetivo, de que tengo unos lentes con los que veo todo oscuro, esa sospecha es preocupante. Porque si fuese así, no importaría qué cambio radical yo haga en mi vida no voy a poder sentirme plenamente satisfecho, contento, feliz.

Acá el consultante que obviamente ya era conciente de que hay perspectivas distintas a la suya, trata de eludir la discusión y nos dice además por qué: porque tiene miedo de que el problema sea él y no el mundo exterior. Y él había dicho que no es posible para él cambiar su perspectiva. Una de las maneras posibles aquí para avanzar en la discusión sería proponer a discusión el dilema entre la posibilidad de un conocimiento universal objetivo y la posibilidad de que sólo exista el limitado conocimiento subjetivo. Esta es la aproximación sugerida por Gruengard, pues parte del supuesto de que en relación a nuestros problemas personales muchas veces no podemos ver con claridad los dilemas filosóficos subyacentes a ellos, y por ello habría que separar la

\footnotetext{
${ }^{16}$ Cuando el consultante dice algo insólito o contradictorio Brenifier frecuentemente plantea al consultante que se imagine qué es lo que dirían otras personas si lo escucharan. El motivo por el cuál hace esto, no es porque se deba suponer que la mayoría tenga razón, sino porque permite al consultante salir de su subjetividad y ponerse en el lugar de otras personas, y darse cuenta de que cambiar de perspectiva es algo que él sí puede y suele hacer. Esta explicación la expone en un a sesión grabada durante su participación en el II Encuentro de Práctica Filosófica en el Perú: BRENIFIER, Oscar: “¿Qué hace que una discusión sea filosófica?” 2da parte, disponible on-line en http://www.youtube.com/watch?v=ey-d_ZhGVww (último acceso 30 de noviembre de 2009)
} 
discusión filosófica de estos dilemas del contexto inicial de donde provenían. Después de aislar el dilema de la situación personal marcada por la subjetividad, podemos contextualizarlo en el marco de discusiones filosóficas de la historia de la filosofía, por ejemplo tomando la discusión entre Platón y Protágoras o Bacon y Hume. La discusión en este plano podría hacernos ver con mayor claridad los presupuestos y consecuencias de las posiciones en disputa y motivaría al consultante a aclarar sus ideas incluso, por analogía, en relación a su situación o problemas personales concretos. También Lahav y Brenifier se remiten a filósofos de la historia de la filosofía, pero no le dan el mismo peso en tiempo y profundidad y detalle que le da Gruengard ${ }^{17}$. Brenifier en estas circunstancias sugeriría señalar las contradicciones o los puntos de vista en disputa del consultante y obligarlo a asumirlos apenas se dan, sin permitir que el consultante evada tomar conciencia de estas contradicciones. En este mismo espíritu, pero sin recurrir en ese momento a la historia de la filosofía, sigo adelante con la discusión.

C.F.: ¿Tú crees que hay personas que ven el mundo sin lentes?

Eduardo: Creo que la lucidez es ver el mundo sin esos lentes oscuros, sin ese sesgo, sin sesgar la realidad.

C.F.: $\quad$ ¿Conoces tú personas que ven el mundo sin lentes, ni oscuros, ni rosados?

Eduardo. No más bien yo veo que la gente tiene unos superlentes. El estado normal de la gente es que tenga una enorme deformación de la realidad que los rodea....

C.F. $\quad$ Esas personas no se preocupan de tener esos lentes. Tú eres el que está preocupado de que esas personas tienen esos lentes.

Eduardo: No, esas personas pueden tener los lentes que quieran. Mi preocupación es que yo pueda tener alguno. Si tuviera uno lentes pegados a mis ojos sería preocupante.

C.F.: $\quad$ ¿Por qué?

Eduardo: Depende de los lentes. Si los lentes me hacen ver las cosas mejor, iqué bueno!, me los quedo.

C.F.: $\quad$ Entonces no te preocupa que deformes la realidad, sólo si es para mal.

Eduardo: Sí, pero preferiría no tener ninguna deformación de la realidad.

C.F.: $\quad$ Tú tienes la esperanza de no tener lentes, y que la realidad sea tan negativa como parece. ¿Por qué te preocupa más que sin

${ }^{17}$ GRUENGARD, Ora, video citado 
lentes pudieras ver la realidad negativa, que la posibilidad de que la realidad sea "objetivamente" positiva, pero que tú no la puedas ver más que negativamente, por tener esos lentes oscuros?

Eduardo se ha cerrado en la posición de que el mundo en su caso personal es "objetivamente" negativo. El prefiere que el mundo esté mal a que en realidad el mundo esté bien y su percepción personal del mundo sea la que sea negativa. Esto parece indicar un presupuesto que se podría explicitar como: "Lo que más importa en la vida es la experiencia subjetiva. Los demás y el mundo no importan." En este punto, lo interesante me pareció que era indagar un poco más en los presupuestos y consecuencias de este postulado. Por ello, me pareció adecuado darle algunas vueltas más a la argumentación. Esto también llevaría a que siente bien su punto de vista, sin necesidad de forzarlo a ello. Si por ejemplo le dijese: "Podríamos decir entonces que: Lo que más importa en tu vida es la experiencia subjetiva. Los demás y el mundo no importan?" Esto podría sonar como una crítica, pues no es lo comúnmente aceptado y el consultante podría tratar de evadir contestar la pregunta dando largas explicaciones. Por otra parte, según el Arte de preguntar de Brenifier podría obligársele a aceptar la hipótesis forzándolo a que conteste con "Sí" o "No" y se vea obligado a asumir las consecuencias de su respuesta. En este caso, no opté por esta aproximación.

Eduardo: Claro... Porque la experiencia que...

C.F.: $\quad$ Te digo por qué te digo eso: Porque podría uno pensar: Si son mis lentes, yo los puedo cambiar y ver la realidad tal cual es, o sea, más positiva; en cambio si realmente la realidad fuese tan negativa, aunque yo me pusiese todos los lentes que quisiera, la verdad sería negativa.

Eduardo: $\quad$ No creo que se pueda cambiar la visión que uno tiene con los lentes.

C.F.: ¿Por qué no?

Eduardo: $\quad$ Porque yo veo que no, que la gente no lo puede cambiar.

C.F.: $\quad$ ¿Te parece que esa es una respuesta?

Eduardo: Jejeje. (...) Pero imagínate que el mundo sea objetivamente maravilloso y que estamos en el mejor de los mundos posibles y que yo lo veo todo negativo por los lentes que no me los puedo arrancar... dado que toda la experiencia es subjetiva, mi experiencia es subjetiva. ¡Qué me importa si el mundo es 
maravilloso si yo lo veo feo! Y además no voy a poder verlo de otra forma

C.F.: $\quad$ Tú piensas que es imposible que puedas cambiar. Y tiene que ver con lo que has dicho: "Se me fue la vida ¿cómo hago para hacer volver la vida?” ¿Cómo cambiar esto? - Tú estás diciendo que no es posible. Porque tú no puedes cambiar.

Eduardo: Pero también es posible que objetivamente el mundo sea negativo y que yo lo estoy viendo claro.

C.F.: $\quad$ Pero lo que más te asusta es que sea subjetivo. Porque tú consideras que no puedes cambiar.... Porque tampoco has cambiado según tú mismo en los últimos 20 años....

Y has preguntado al inicio: “¿Por qué no lo he hecho?” y la respuesta sería: Porque tú piensas que no se puede.

Esta propuesta de conclusión tiene como propósito, por una parte, relacionar las partes de lo dicho en la sesión y por otra parte, sugerir al consultante que proponga una alternativa a este aparente círculo vicioso en su pensamiento: No lo he hecho porque no se puede - La prueba de que no se puede hacer es que no lo he podido hacer o no lo ha podido hacer nadie.

A estas alturas conviene hablar sobre cuál considero yo que es la finalidad de esta dinámica y de una consultoría filosófica. Cabe recordar que ya al principio mencioné que independientemente de cual sea nuestra fundamentación teórica de la consultoría filosófica y la práctica filosófica en general, en última instancia, el resultado, esto es, la práctica concreta tiene cierta independencia con respecto a nuestro propósito teórico y justamente por eso, es que es posible que prácticas concretas de consultoría filosófica que parten de presupuestos muy diferentes como los de Lahav, Brenifier y Gruengard, a los ojos de observadores externos, coincidan en algunos puntos en la práctica concreta.

En mi caso, parto del presupuesto teórico de que tendemos a adaptar nuestras teorías a la práctica y no al revés. En otras palabras: lo que hacemos es lo que realmente nos define, y si nuestro discurso a veces se contradice con nuestra práctica, la tendencia es que al final vamos a justificar nuestras acciones y actitudes adaptando nuestro discurso a nuestra práctica y no adaptando nuestras acciones y actitudes a nuestro discurso. 
En ese sentido, por una parte, nos ejercitamos en el análisis y ordenamiento coherente de nuestro discurso, pero en última instancia lo importante es reflexionar sobre las acciones y actitudes del consultante, y sobre los presupuestos filosóficos que se esconden detrás de estas acciones y actitudes. El instrumento del cual nos valemos para reflexionar conjuntamente sobre estas acciones y actitudes es el discurso y en especial el diálogo.

Por eso, dejo que el consultante desarrolle su perspectiva y se enfrente teóricamente a las contradicciones que se puedan desprender de ella, para luego pasar a lo más importante, que es la confrontación de lo discutido con una reflexión sobre las acciones en la vida concreta del consultante.

La consultoría filosófica desde esta perspectiva es entonces un ejercicio de pensamiento sobre el hombre dirigido por un filósofo, pasando por la reflexión filosófica sobre el individuo particular, tomando en cuenta tanto las acciones y actitudes en su vida en general, como las acciones y actitudes reflejadas durante la misma sesión de reflexión filosófica.

Estos presupuestos de la práctica filosófica se distinguen un poco de los de Lahav por el peso que le doy al interés epistemológico, en el sentido de que considero al consultante y a la dinámica con el consultante, como objetos de estudio.

Para Lahav ${ }^{18}$ el objetivo primario de los consultores filosóficos es ayudar a crecer al consultante haciéndolo conciente de los presupuestos filosóficos que subyacen a sus actitudes, emociones y acciones, y a través de la comprensión de las limitaciones que resultan de nuestros patrones de comportamiento habituales. Esta práctica la he recogido de Lahav quien nos introdujo a ella por los talleres de consultoría filosófica individual y grupal que hemos tenido con él, así como el seguimiento y

\footnotetext{
18 "Como filó-sofos prácticos nuestro objetivo primario es crecer a través de la comprensión, no es fijar comportamientos o emociones. Entendemos las enormes fuerzas que sostienen nuestros patrones y concepciones. Nos damos cuenta de que nuestra fuerza de voluntad y autocontrol tienen poca capacidad de cambiarnos. Como consejeros o coordinadores de talleres abandonamos la esperanza ingenua de que el auto-conocimiento le permita al aconsejado cambiar de alguna manera sustancial. Tenemos entendido, que aun cuando los consultantes están concientes de sus patrones e incluso cuando deciden cambiarlos, la distancia a un cambio efectivo es enorme." LAHAV, Ran: "Sobre las fuerzas" en http://www.trans-sophia.net/115845/6--Sobre-las-fuerzas (último acceso 30 de noviembre de 2009)
} 
discusiones de consultorías filosóficas supervisadas en un primer momento. La consultoría filosófica sin embargo, para Lahav, no debe considerar al consultante como objeto de estudio, sino que si bien es un paso de investigación hacia dentro de nosotros mismos, debería servir sólo como un peldaño para continuar luego más allá del ámbito de la filosofía y más allá de las limitaciones del pensamiento analítico, para abrirnos a horizontes de vida más amplios, lo que Lahav llama la vía más allá de la filosofía:"trans-sofía" 19 .

Brenifier por su parte, ${ }^{20}$ parte del presupuesto de que la filosofía es una actividad inherente al ser humano y que como seres humanos compartimos un mundo común y una misma condición, por lo que es posible encontrar en cada uno de nosotros algo de "un número determinado de los arquetipos intelectuales que constituye el armazón de la historia del pensamiento" y de la filosofía. El objetivo de la consultoría filosófica para Brenifier es evidenciar a través de la mayéutica los diferentes presupuestos filosóficos contradictorios en cada uno de nosotros, que de alguna manera son el reflejo y reflejan las diferentes posiciones filosóficas a lo largo de la historia del pensamiento. Para ello no toma en cuenta solo el discurso del consultante, sino también sus

\footnotetext{
${ }^{19}$ LAHAV, Ran: "Trans-Sophia - Filosofía espiritual - Práctica filosófica y más allá”, disponible on-line en:
}

http://www.trans-sophia.net/site/index.asp?depart_id=115845\&lat=es (último acceso $30 \mathrm{de}$ noviembre de 2009)

20 "Como seres humanos, compartimos un mundo común (a pesar de la infinidad de las representaciones que cada uno de nosotros experimentamos) y una misma condición -o naturaleza- (a pesar del relativismo cultural e individual que nos rodea), por lo que deberíamos ser capaces de encontrar, al menos de manera embrionaria, un número determinado de arquetipos intelectuales que constituyesen el armazón de la historia del pensamiento. Si después de todo, la fuerza de una idea descansa sobre su operatividad y su universalidad, cada una de esas "ideas fuerza" debería poder encontrarse en cada uno de nosotros. ¿Acaso no es ésa la idea misma de la reminiscencia platónica, aunque formulada en otros términos y desde otra perspectiva? (...) De este modo, nuestro ejercicio consiste en primer lugar en que el sujeto identifique, a través de sus opiniones, los presupuestos inconfesables con los que suele funcionar, lo que permite definir claramente los puntos de partida. En segundo lugar, sirve para tener en cuenta la hipótesis contraria a estos presupuestos, con el fin de transformarlos de indiscutibles postulados en simples hipótesis. En tercer lugar, el sujeto deberá articular las problemáticas así generadas a través de conceptos identificados y formulados. En esta última etapa -o incluso antes si la utilidad así lo demanda-, el orientador filosófico podrá utilizar las problemáticas "clásicas", propias de un autor determinado, con el fin de valorar o identificar mejor este o aquel aspecto que surjan en el transcurso de la consulta." BRENIFIER, Oscar: "La consulta filosófica - los principios", disponible en:

http://www.brenifier.com/espanol/orienta_principios.htm (último acceso 30 de noviembre de 2009) 
actitudes durante la consultoría, pues estas afirman determinadas posiciones y valores, de las que el consultante muchas veces no es plenamente conciente. A través de este ejercicio mayéutico Brenifier también facilita la toma de conciencia de las trabas que han impedido evidenciar al consultante los presupuestos filosóficos en su vida cotidiana.

Quiero notar que usamos vocabularios diferentes para referimos a contenidos bastante similares. Al igual que Brenifier, también Gruengard habla de la importancia de encontrar las oposiciones principales que subyacen a las preocupaciones del consultante. Específicamente dice que la consultoría filosófica está basada en la asunción de que "las dificultades para tratar los problemas tienen que ver a menudo con una inhabilidad para resolver o disolver los dilemas filosóficos subyacentes"21 y que por lo tanto la "de-construcción" de los problemas del aconsejado en dos grupos de oposiciones y su reconstrucción en dilemas filosóficos puede ayudarlo a tratar con los problemas de su mundo de una manera constructiva y productiva. En este sentido se diferencia de Lahav y Brenifier, pues piensa que una vez determinado el dilema filosófico principal que subyace a su vida o al problema planteado, es aconsejable distanciarse del problema concreto y desentrañar y profundizar la reflexión teórica sobre el dilema filosófico recurriendo también a la historia de la filosofía o del pensamiento universal para ampliar la perspectiva del dilema planteado, para luego retornar y por analogía desentrañar el dilema de la situación concreta personal.

En resumidas cuentas, los puntos de partida y propósitos de estos (y otros) consultores filosóficos varían bastante, aunque no precisan, ni detallan de manera sistemática sus perspectivas y aproximaciones, porque consideran que sería una forma de encasillar su quehacer. Por ello no es posible hacer una comparación real entre ellos, sino que los presento

\footnotetext{
21 “Mi aproximación a la consejería filosófica está basada en la asunción de que las dificultades `para tratar los problemas tienen que ver a menudo con una inhabilidad para resolver o disolver los dilemas filosóficos subyacentes. A este respecto, no hay diferencia entre los problemas teóricos y prácticos. Por lo tanto el estar familiarizado tanto de la historia de los problemas teoréticos y los procesos por los cuales ellos fueron resueltos y disueltos, vencidos, pasados por, o, de lo contrario, vueltos a emerger-- puede ser una fuente de ideas y herramientas que son aplicables en casos prácticos también."

GRUENGARD, Ora: Conocimiento y Diálogo en la Consejeria Filosófica, ob.cit
} 
paralelamente para mostrar algunas diferencias en los matices de sus aproximaciones.

\section{Sobre la relación que el dilema planteado en teoría tiene con la práctica concreta del consultante}
Eduardo:
Sí, esto es un caso trágico
C.F.:
Claro, ni tú puedes cambiar, según tú, ni tampoco puedes cambiar tú el mundo, según tú...
Eduardo: Conclusión: Todo se va a quedar igual.
C.F.: $\quad$ Eso responde a tu pregunta: “¿Por qué no lo he hecho?” Ya está resuelta tu pregunta.
Eduardo: Eso puede calmar la inquietud, pero deja igual una situación de desagrado....
C.F.: $\quad$ Fíjate que al inicio habías introducido el factor de la edad, o sea la vejez.
Eduardo: Ah sí...
C.F.: $\quad$ Si tú no puedes cambiar tú subjetivamente, ni tampoco puedes cambiar el mundo, eso no lo puedes hacer a ninguna edad, ni de niño, ni de adolescente, ni de joven, ni de viejo, según lo que estás postulando.

Propongo una conexión entre la conversación que tuvimos sobre realidad objetiva y percepción subjetiva y el problema planteado inicialmente sobre que el consultante estaría ya muy viejo para cambiar su vida. Ahora el trabajo consiste en volver a la realidad concreta del consultante.

Eduardo: Sí pues... Si, pero es igual que el ejemplo de la isla desierta, por ejemplo, como alguien que...

C.F.: $\quad$ Siempre pones ejemplos extraños, no pones ejemplos de ti mismo... Da un ejemplo de una situación donde hayas querido cambiar algo, y no resultó.... Ya sea a ti mismo o a algo externo.

Inducir al consultante a precisar lo que quiere contando una experiencia concreta de su vida, y si es posible un hecho reciente, en el que ha sentido o ha ocurrido aquello a lo que hace referencia es una buena manera de entender con más precisión lo que quiere decir y al mismo tiempo penetrar el contexto en el que el problema se está planteando. Es un paso 
que aprendí a usar en los talleres de Lahav ${ }^{22}$, pero que también es usado por Gruengard. ${ }^{23}$

Eduardo: Puede ser por ejemplo, cuando quería que acaben los comentarios homófobos en el Buho (Café Filosófico) ... yo veo la diferencia entre cuando empezó todo eso hace meses...y hubo todo eso.. De ese momento, y ahora.....Ahora ya no lo haría.... La defensa siempre tiene que ver con la edad.... Si hubiera sabido que más o menos todo iba a seguir igual, no hubiera hecho nada..

C.F.: ¿Qué cosa es lo que no ha cambiado?

Eduardo: $\quad$ O sea, tú me has pedido que te de un ejemplo de que no se puede cambiar el mundo.

C.F.: $\quad$ Claro...

Finalmente el consultante introdujo el tema sobre los Cafés Filosóficos que me involucra. El contexto de la situación es que con unos amigos filósofos dirigimos un Café Filosófico al cual ha venido asistiendo un buen tiempo. En algún momento él protestó porque habían participantes que hacían alusiones o chistes homófobos durante sus intervenciones y después de una serie de percances, finalmente la directiva, de la cual formo parte, publicó un nuevo reglamento que explícitamente prohibía hacer este tipo de alusiones, a riesgo de ser expulsado del Café. En el contexto de estos cambios en el reglamento el consultante había hecho una denuncia pública por internet acusando a nuestro equipo de ser homofóbico.

Este momento es interesante porque muestra que también hay un factor psicológico en la interacción entre las partes, al cual, como se dijo al inicio, no debe permitírsele interferir en la sesión. Este factor también puede ser difícil de manejar cuando se habla de sexualidad y atracción sexual entre las partes, $u$ otras situaciones donde el consultante involucra a la persona del consultor en la discusión.

Y así, a pesar de que pregunto una pregunta objetiva ("¿Qué cosa es lo que no ha cambiado?") con la intención de que el consultante precise

\footnotetext{
${ }^{22}$ LAHAV, Ran: "Practica Filosófica Grupal con Ran Lahav 1 ( de 12)" disponible en: http://video.google.de/videoplay?docid=-9053698290533005892\# (último acceso 30 de noviembre de 2009)

${ }^{23}$ GRUENGARD, Ora, video citado
} 
lo que está queriendo decir, el consultante reacciona a la pregunta a la defensiva como si le estuviera pidiendo aclaraciones.

Eduardo: Yo compruebo que antes pensaba que sí (se puede cambiar el mundo). Yo pensaba que pataleando, rabiando, haciendo escándalo, pensaba que al final de todo eso podía empezar a cambiar algo...

C.F.: ¿Tú pensabas que logrando que los directivos del Buho (institución en la que se realizan los Cafés Filosóficos) establezcan que no se puede hacer comentarios homófobos, y que eso además estuviera explícito puesto sobre papel, eso podría hacer que la gente, los asistentes, en su mayoría no fuesen homófobos? O no hicieran declaraciones homófobas... ¿Y eso según tú no se ha cumplido..? Eso no se cambió...

Eduardo: Allí volvemos a la antigua preocupación. Yo creo que sí hay un cambio, pero ahora ya no me importa mucho... o que no me parece tan positivo...

Acá notamos por qué es fundamental pasar al análisis de los hechos concretos. Según su discurso el consultante afirmaba que es imposible cambiar él mismo o cambiar situaciones del mundo externo. Sin embargo, al dar un ejemplo concreto de un intento fallido de cambio, tanto el consultor como el consultante mismo notan, que el dilema no era que no se pudiese cambiar en nada al mundo externo, sino que al producirse el cambio deseado se daba otro fenómeno: que el objetivo deseado dejaba de ser deseado.

C.F.: $\quad$ Fíjate que hace un rato tú dijiste que habían esas personas que luchaban por un cambio, y cuando lo conseguían, ya no les interesaba y eso era una de las pruebas de que tenían unos lentes que le hacían ver las cosas negativamente.

Eduardo: Sí, puede ser que yo también tenga esos lentes. Sí, yo creo que la cosa ha mejorado, pero como que no....Tenía otra teoría aparte de la de los lentes y que también la he estado pensando esta semana.. Era la idea de que uno sólo es feliz cuando uno necesita algo... cuando te falta... no cuando tienes.... 
Ante la evidencia para el consultante, de que él es justamente tal como él había criticado que eran los otros, el consultante cambia de tema. Tanto Brenifier, como Lahav y Gruengard señalan que cuando el consultante cambia abruptamente de tema, es porque hay algo importante allí que trata de eludir y sobre lo cual se podría seguir trabajando. Esta observación sobre el cambio de tema podríamos calificarla de psicológica y podría decirse que se sale del ámbito de la filosofía. Sin embargo, al analizar o tomar en cuenta las actitudes y reacciones del consultante, el consultor no busca sus causas psicológicas sino los presupuestos filosóficos subyacentes. Tales como por ejemplo pudieran ser: "El mundo es negativo. El individuo no puede hacer nada para cambiar su visión del mundo. Aunque cambie algo en su mundo, su visión seguirá siendo negativa." Por otra parte como dice Lahav, las acciones y actitudes de una persona no son psicológicas, ni filosóficas, sino hechos concretos. Y es sobre estos hechos que la práctica filosófica centra su atención.

C.F.: Cuéntame una situación en la que te sentías feliz porque te faltaba algo...

Eduardo: Por ejemplo con mi pareja, cuando estaba con ella tenía la permanente necesidad de estar con ella.

En este caso estimé que si el consultante quería cambiar de tema, lo hiciera. De alguna manera de todos modos ya se había dado cuenta del dilema subyacente al problema que planteó. Y también quedó develada la contradicción entre lo que postuló inicialmente en el sentido de que no habría hecho nada de lo que quiso hacer en su vida, y la constatación al dar ejemplos de su vida, de que sí había hecho cosas que quiso. Se mostró que el problema era otro, el de la pérdida de interés en las metas logradas. Y así pasamos a desarrollar el tema de la felicidad, como una condición ligada a la sensación de que a uno le falta algo, que necesita algo.

\section{Conclusiones de la sesión}

C.F.: $\quad$ Vamos a parar ahora, porque se nos ha acabado el tiempo... ¿Qué puntos piensas que se te han aclarado a partir de lo que hemos estado hablando- o tal vez ninguno-? 
Eduardo: $\quad$ Se ha hecho un poco más claro el problema, lo veo un poco más claro el problema. Parece que hubiese más personajes en el tramado del problema. Pero no veo clara la solución.

C.F: $\quad$ ¿Cuáles personajes hay en el tramado del problema.

Eduardo: Uno de esos personajes son los lentes, y la posibilidad de que existan, el otro podría ser esto de la felicidad como deseo, como momento de plenitud. Y todo eso tiene relaciones, pero todavía no entiendo bien las relaciones

Tanto Brenifier, como Lahav y Gruengard coinciden en la necesidad de dejar hacer una evaluación final y/o un resumen final al consultante, en el que deseable es hacer repreguntas cuando las respuestas son muy vagas como en este caso. También es muy común que el consultante esté algo agotado y confundido por la intensa presión del ejercicio del pensar durante una hora, y en ese sentido una mayor claridad de lo pensado a veces recién se produce en las horas o días subsiguientes al ejercicio, en los que va reflexionando sobre lo dicho y estableciendo conexiones.

\section{Conclusión y propuesta}

A través del análisis de esta consultoría he tratado de mostrar similitudes y diferencias en el trabajo práctico concreto de la consultoría filosófica. Ha resultado que más son las coincidencias que las diferencias. He presentado también los diferentes puntos de vista sobre qué es la práctica filosófica, de la que parto yo y de la que parten Lahav, Brenifier y Gruengard respectivamente. La intención de este artículo sin embargo no era entrar en discusión sobre cuál teoría o cual práctica es la más pertinente, ni siquiera es discutir el contenido de esta sesión escogida al azar para su análisis, sino es mostrar cómo en la práctica hay pasos que se asemejan a pesar de partir de posiciones filosóficas distintas. Y hemos tratado de mostrar cómo este se debe principalmente a que en la práctica concreta el consejero filosófico se tiene que adaptar a las circunstancias concretas del mundo del consultante, más que en sus presupuestos teóricos.

Propongo aquí, que al igual que se puede sistematizar cómo se debe articular un texto filosófico para su publicación, se pueden sistematizar 
ciertos pasos en la forma de llevar a cabo la consultoría filosófica., como por ejemplo formas de iniciar la sesión, modos de preguntar y de repreguntar, maneras de hacer que el consultante conecte su discurso con experiencias concretas de su realidad, modos de continuar en caso de entrampamiento, etc. ${ }^{24}$ La sistematización de la forma, no significaría la sistematización, ni la uniformización de los contenidos de la consultoría filosófica, de la misma manera que un artículo filosófico bien articulado no significa una uniformización de los contenidos. Es más, pueden haber diferentes formas de hacer dinámicas en la consultoría filosófica, y todas ellas pueden ser sistematizadas. De hecho en los talleres de práctica filosófica de alguna manera está sobreentendido que las dinámicas y/o técnicas para el diálogo con el consultante pueden sistematizarse. Uno de los motivos principales por los cuales ha habido una reticencia a plasmar esta sistematización por escrito, es que se teme que se podría desvirtuar la práctica filosófica, convirtiéndola en una mera técnica. Pero eso sería igual que afirmar que no debe haber una estructura en un artículo filosófico, porque se desvirtuaría su contenido filosófico. El contenido filosófico de una práctica o de un texto filosófico no está principalmente en su forma, sino en su contenido. Y por último, el hecho de que los textos filosóficos tengan normalmente una determinada estructura, no impide que haya filósofos como Nietzsche o Platón, que se salgan del esquema habitual, así como cierta sistematización de los pasos en práctica filosófica tampoco impide que se experimente con otras formas de hacer práctica filosófica.

La utilidad de explicitar la forma en el trabajo de la práctica filosófica, en este caso, de la consultoría filosófica es por una parte facilitar su difusión y reconocimiento como un quehacer filosófico, y por otra parte, explicitar pasos que no se deben hacer, tales como dar consejos al consultado sobre lo que debe hacer o no hacer, o hacer una crítica desde determinada posición ética de lo que ha dicho o ha hecho el consultado, y otros pasos que impiden que se de el momento de reflexión

\footnotetext{
${ }^{24}$ Brenifier ha trabajado algo en este sentido. En su libro Diálogo en clase, Tenerife: Idea, 2005,pp. 123-131 en el capítulo 8 "Obstáculos y estrategias de resolución” trata de sistematizar las dificultades mas frecuentes que se presentan durante la discusión en la práctica filosófica y propone estrategias de resolución.
} 
filosófica por parte del consultado y que por lo tanto desvirtúan la consultoría filosófica.

En este artículo no estoy proponiendo aun una sistematización de la forma, sólo indico la utilidad de hacerlo. Para ello sería bueno recopilar técnicas y experiencias de diferentes filósofos prácticos de manera sistematizada.

La ventaja de la sistematización de la forma es que facilita que como filósofos nos centremos más en el contenido, es decir, podamos centrarnos más en profundizar la reflexión filosófica durante la sesión, pero también en recoger lo discutido y plasmarlo en documentos tanto metafilosóficos, para profundizar sobre lo ocurrido en la sesión, como en una producción filosófica inspirada en estos momentos de discusión o reflexión filosóficos. Como precursores de este tipo de documentos podrían considerarse a la Historia de mis calamidades $^{25}$ de Pedro Abelardo y los diálogos de Sócrates con los intelectuales y políticos de su época repensados por Platón ${ }^{26}$, respectivamente. Estos ejemplos, entre otros, nos muestran que el rigor metodológico en las discusiones filosóficas, no impide que su reflexión posterior sobre ellas (Abelardo) o su elaboración a formas más inspiradoras (Platón), sea de carácter más libre y profundamente filosóficas. Y tampoco hay que suponer que los diálogos que Sócrates llevara a cabo con sus diferentes interlocutores no fueran de carácter filosófico y/o que lo filosófico sólo lo constituiría la forma de presentación de estos diálogos por parte de Platón en sus Diálogos.

Algunos filósofos prácticos han empezado a trabajar en esta dirección. Estoy pensando en las historias que Lahav cuenta en sus Voces de la Realidad Humana ${ }^{27}$ y en textos tales como El amor - la locura $\operatorname{divina}^{28}$ de Achenbach, entre otros que deben existir y muchos otros

\footnotetext{
${ }^{25}$ ABELARDO, Pedro: Historia de mis Calamidades, Buenos Aires: Centro Editor de América Latina, 1983

${ }^{26}$ PLATON: Diálogos, Madrid: Gredos, 1992- 2002

${ }^{27}$ LAHAV, Ran, "Voces de la Realidad Humana" disponible en: http://www.trans-sophia.net/?lat=es (último acceso 30 de noviembre de 2009)

28 ACHENBACH, Gerd: Liebe - der göttliche Wahn, Freiburg: Herder, 2006. En este libro Achenbach en una analogía con el diálogo el Fedro de Platón a través de los personajes Goodmann, el filósofo, la médico, entro otros, reproduce una serie de discusiones sobre el amor, que parecen estar inspiradas en discusiones de sus reuniones filosóficas y de alguna que otra consultoría filosófica.
} 
textos y materiales audiovisuales que esperamos que no tardarán en aparecer.

\section{Bibliografía}

ACHENBACH, Gerd: “Al centro del la Práctica Filosófica” (Zur Mitte der philosophischen Praxis, traducción propia), Conferencia de apertura del II Congreso Internacional de Práctica Filosófica en Amersfoort/Leusden, Holanda, 25 de agosto de 199, accesible en http://www.achenbachpp.de/cont/vortraege_pp.asp

- "El arte de saber conversar", Ponencia para el 20 aniversario de la "Sociedad para la conducción de conversaciones en idiolecto" (GIG), Würzburg, 5 de mayo del 2005, accesible en http://www.achenbach-pp.de/cont/vortraege_pp.asp (último acceso 30 de noviembre de 2009)

- Liebe - der göttliche Wahn (El amor - la locura divina), Freiburg: Herder, 2006.

ABELARDO, Pedro: Historia de mis Calamidades, Buenos Aires: Centro Editor de América Latina, 1983

BRENIFIER, Oscar: Diálogo en clase, Tenerife: Idea, 2005

- "El discurso racional no puede llegar a ser un instrumento para abordar todos los problemas del hombre" disponible on-line en :

http://www.brenifier.com/espanol/videos/el_discurso_raciona 1_no_puede_llegar.htm (último acceso 30 de noviembre de 2009)

- "Filosofar es dejar de vivir", disponible on-line en www.brenifier.com/espanol/filosofar_es_dejar_de_vivir.htm (último acceso 30 de noviembre de 2009).

- "La consulta filosófica - los principios", disponible en: http://www.brenifier.com/espanol/orienta_principios.htm (último acceso 30 de noviembre de 2009) 
- “¿Qué hace que una discusión sea filosófica?” 2da parte, disponible on-line en http://www.youtube.com/watch?v=eyd_ZhGVww (último acceso 30 de noviembre de 2009)

GRUENGARD, Ora: "Conocimiento y Diálogo en la Consejería Filosófica", Conferencia presentada en el "I Encuentro de Práctica Filosófica en el Perú" información disponible online en http://www.redfilosofica.de/practicafilosoficainternacional/

- "Ora Gruengard: Consultoría Filosófica (muestra)" disponible on-line en en video.google.de/videoplay?docid=6060796800723049099\&\# (último acceso 30 de noviembre de 2009)

LAHAV, Ran: "Curso en Práctica Filosófica" disponible on-line en http://www.trans-sophia.net/115845/7--E-La-exploraci-n-delper-metro (último acceso 30 de noviembre de 2009)

- "Desarrollando la Sensibilidad Filosófica", disponible on-line en www.trans-sophia.net/115845/21--La-sensibilidad (último acceso 30 de noviembre de 2009)

- "El concepto de perímetro" disponible on-line en http://www.trans-sophia.net/115845/1--El-Concepto-de-Permetro (último acceso 30 de noviembre de 2009)

- "Practica Filosófica Grupal con Ran Lahav 1 ( de 12)" disponible en:

http://video.google.de/videoplay?docid=-

9053698290533005892\# (último acceso 30 de noviembre de 2009)

- "Ran Lahav en Consultoría Filosófica con Carmen (Inglés con subtítulos en español)" disponible on-line en http://www.trans-sophia.net/115845/VIDEO--Ran-Lahav-enConsultaria-Filosofica-con-Carmen (último acceso 30 de noviembre de 2009)

- "Sobre las fuerzas" en http://www.transsophia.net/115845/6--Sobre-las-fuerzas (último acceso 30 de noviembre de 2009) 
- “Trans-Sophia - Filosofía espiritual - Práctica filosófica y más allá", disponible on-line en: http://www.transsophia.net/site/index.asp?depart_id=115845\&lat=es (último acceso 30 de noviembre de 2009)

- "Voces de la Realidad Humana" disponible en: http://www.trans-sophia.net/?lat=es (último acceso 30 de noviembre de 2009)

MARX, Karl: “Tesis sobre Feuerbach", Tesis 11 en: Obras escogidas, T I, Moscú: Editorial Progreso, 1976

PLATON: Diálogos, Madrid: Gredos, 1992- 2002 
